\title{
Bipolar disorder with binge eating behavior: a genome-wide association study implicates PRR5-ARHGAP8
}

\author{
Susan L. McElroy ${ }^{1,2}$, Stacey J. Winham³ ${ }^{3}$ Alfredo B. Cuellar-Barboza ${ }^{4}$, Colin L. Colby ${ }^{3}$, Ada Man-Choi Ho $\mathbb{1}^{5}$, \\ Hugues Sicotte ${ }^{3}$, Beth R. Larrabee ${ }^{3}$, Scott Crow ${ }^{6}$, Mark A. Frye ${ }^{7}$ and Joanna M. Biernacka $\mathbb{1}^{3,7}$
}

\begin{abstract}
Bipolar disorder (BD) is associated with binge eating behavior (BE), and both conditions are heritable. Previously, using data from the Genetic Association Information Network (GAIN) study of BD, we performed genome-wide association (GWA) analyses of BD with BE comorbidity. Here, utilizing data from the Mayo Clinic BD Biobank (969 BD cases, 777 controls), we performed a GWA analysis of a BD subtype defined by BE, and case-only analysis comparing BD subjects with and without BE. We then performed a meta-analysis of the Mayo and GAIN results. The meta-analysis provided genome-wide significant evidence of association between single nucleotide polymorphisms (SNPS) in PRR5-ARHGAP8 and $\mathrm{BE}$ in $\mathrm{BD}$ cases (rs726170 OR=1.91,P=3.05E-08). In the meta-analysis comparing cases with BD with comorbid BE vs. non-BD controls, a genome-wide significant association was observed at SNP rs111940429 in an intergenic region near PPP1R2P5 $(p=1.21 \mathrm{E}-08)$. PRR5-ARHGAP8 is a read-through transcript resulting in a fusion protein of PRR5 and ARHGAP8. PRR5 encodes a subunit of $\mathrm{mTORC2}$, a serine/threonine kinase that participates in food intake regulation, while ARHGAP8 encodes a member of the RhoGAP family of proteins that mediate cross-talk between Rho GTPases and other signaling pathways. Without $\mathrm{BE}$ information in controls, it is not possible to determine whether the observed association reflects a risk factor for $\mathrm{BE}$ in general, risk for $\mathrm{BE}$ in individuals with $\mathrm{BD}$, or risk of a subtype of $\mathrm{BD}$ with $\mathrm{BE}$. The effect of PRR5-ARHGAP8 on BE risk thus warrants further investigation.
\end{abstract}

\section{Introduction}

Bipolar disorder $(\mathrm{BD})$ and binge eating behavior $(\mathrm{BE})$ co-occur more often than expected by chance ${ }^{1-3}$. Estimated to occur in $4.5 \%$ of the United States general population, BE has been reported to occur in over $25 \%$ of individuals with $\mathrm{BD}^{1,2}$. Defined as eating an unusually large amount of food in a discrete time period with a sense of loss of control over the eating, BE is a transdiagnostic feature of eating disorders: it is a defining symptom of bulimia nervosa and binge eating disorder,

Correspondence: Joanna M Biernacka (biernacka.joanna@mayo.edu) ${ }^{1}$ Lindner Center of HOPE, Mason, OH, USA

${ }^{2}$ Department of Psychiatry and Behavioral Neuroscience, University of Cincinnati, Cincinnati, $\mathrm{OH}$, USA

Full list of author information is available at the end of the article Susan L. McElroy and Stacey J. Winham contributed equally to this work. and may also occur in anorexia nervosa. BD with comorbid $\mathrm{BE}$ is associated with greater psychiatric and general medical burden than $\mathrm{BD}$ without $\mathrm{BE}$, and has been proposed to be an important clinical BD subtype ${ }^{4}$. Additionally, each condition is heritable, with heritability estimated at $60-85 \%$ for $\mathrm{BD}$ and $46-74 \%$ for $\mathrm{BE}^{5-9}$. Indeed, parental $\mathrm{BD}$ is a risk factor for eating disorders, including those with $\mathrm{BE}$, in offspring ${ }^{10}$. Few studies, however, have evaluated the genetic architecture of the co-occurrence of BD and BE. Genetic studies of specific $\mathrm{BD}$ sub-phenotypes may help uncover some of the unknown inheritance of BD. In an earlier study using data from the Genetic Association Information Network (GAIN) study of BD, we conducted a genome-wide association (GWA) analysis of BD subtypes defined by the presence or absence of a history of $\mathrm{BE}$ and a case-only 
analysis comparing $\mathrm{BD}$ subjects with and without $\mathrm{BE}$ history ${ }^{11}$. In that study, no associations were statistically significant at the genome-wide level.

In this study we perform similar analyses in an independent sample of BD patients from the Mayo Clinic Biobank, use the new results to evaluate replication of the prior GAIN results, and conduct a meta-analysis of the two studies, making this the largest GWA study (GWAS) of BE in BD to date. To determine if the GWAS results were enriched for biologically relevant pathways, we then conduct gene-set and network analysis. We also explore specific gene regions previously implicated in $\mathrm{BE}$ or related phenotypes.

\section{Methods}

\section{Study participants from GAIN}

We previously performed GWA analyses of BE using data from BD cases and controls collected by the Bipolar Disorder Genome Study Consortium, part of GAIN ${ }^{11}$, accessed using $\mathrm{dbGaP}^{12}$. Subjects included 1001 European American bipolar cases and 1034 mentally healthy European American controls. BD cases met DSM-IV criteria for bipolar I disorder or schizoaffective disorder determined by psychiatric interviews based on the Diagnostic Interview for Genetic Studies (DIGS) 2, 3, or 4, which included the question, "has there ever been a time in your life when you went on food binges (i.e., rapid consumption of a large amount of food in a discrete period of time, usually less than two hours)?". Based on answering this question (yes/no), $\mathrm{BD}$ cases were classified as having $\mathrm{BE}$ $(N=206)$ or not having BE $(N=723)$. BE data was not available for controls.

\section{Study participants from Mayo Clinic Biobank}

Here, a new sample of BD cases from the Mayo Clinic Bipolar Disorder Biobank ${ }^{13}$, a collaboration between Mayo Clinic and the Lindner Center of HOPE, and controls from the Mayo Clinic Biobank ${ }^{14}$, was used for GWA analyses of BE. Both biobank protocols were approved by an institutional review board, and every participant provided written informed consent to be included in the biobank and future genetic studies. All subjects from the Mayo Bipolar Disorder Biobank had a diagnosis of bipolar I disorder, bipolar II disorder or schizoaffective bipolar type. Entry criteria included age 18 through 80 years and no current suicidal ideation or psychosis. The clinical phenotype of $\mathrm{BD}$ subjects was determined with the Structured Clinical Interview for DSM-IV $(\text { SCID })^{15}$ and the Bipolar Biobank Clinical Questionnaire. Current BE was assessed with the Eating Disorder Diagnostic Scale $(\text { EDDS })^{16-18}$. EDDS data was available for 700 of the BD cases passing genotype QC. For the BD plus BE phenotype, subjects had to answer yes on questions 5 and 6 of the EDDS ["During the past 6 months have there been times when you felt you have eaten what other people would regard as an unusually large amount of food (e.g. a quart of ice cream) given the circumstances?" and "During the times when you ate an unusually large amount of food, did you experience a loss of control (feel you couldn't stop eating or control what or how much you were eating)?"], and thus have any BE within the past six months ( $N=192$ of $700 \mathrm{BD}$ cases with EDDS data).

The control group was selected from the Mayo Clinic Biobank $^{14}$, and excluded potential controls with ICD9 codes for bipolar disorder, schizophrenia, or related diagnoses in their electronic medical record. BE was not assessed among the controls.

\section{Genotyping, quality control, and imputation}

GAIN samples were genotyped on the Affymetrix Genome-Wide Human SNP Array 6.0. Quality control (QC) was performed as described previously ${ }^{11,19}$. After QC, the sample included 1001 European American BD cases and 1034 mentally healthy controls.

Mayo cases and controls (total $N=1864$ ) were genotyped using the Illumina HumanOmniExpress platform. We removed SNPs with call rate $<98 \%$, minor allele frequency $<0.01$, and those demonstrating departures from Hardy-Weinberg Equilibrium $(P<1 \mathrm{e}-06)$. We excluded subjects with $<98 \%$ call rate, one subject from each related pair (estimated identical-by-descent allele sharing $>.2$ ), and subjects of non-European ancestry. We used Structure ${ }^{20}$ to verify European ancestry, and adjusted for remaining population structure using the first four principle components. After QC, the Mayo sample consisted of 969 BD cases and 777 healthy controls without BD.

Genotypes in both the GAIN and Mayo samples were imputed to the 1000 genomes reference panel, as previously described for the GAIN sample ${ }^{11}$. Specifically, SHAPEIT $^{21}$ was used for haplotype phasing, followed by imputation using IMPUTE2.2.2 $2^{22}$ with the 1000 genome project reference data (phase 1 data, all populations). SNPs with dosage $\mathrm{R}^{2}<0.3$ or MAF $<0.01$ were removed prior to analysis, resulting in more than 8 million SNPs in both datasets.

\section{Statistical analysis}

To identify genetic risk variants of $B E$ in subjects with $\mathrm{BD}$, we previously performed a GWAS of BE in BD GAIN subjects (206 BD subjects with BE vs. 723 BD subjects with no $\mathrm{BE})^{11}$. We now performed a similar GWAS of BE using the Mayo sample (192 BD subjects with BE vs. 508 $\mathrm{BD}$ subjects with no $\mathrm{BE}$ ) and then conducted a metaanalysis of the two studies.

To identify genetic risk variants for a subtype of BD with $\mathrm{BE}$, we also performed a GWAS of BD with comorbid BE, comparing $\mathrm{BD}$ cases with $\mathrm{BE}$ vs. non-bipolar controls in 
both the GAIN ${ }^{11}$ (206 BD subjects with BE vs. 1034 nonBD controls) and Mayo (192 BD subjects with BE vs. 777 non-BD controls) samples, and then conducted a metaanalysis of the results.

In each dataset, logistic regression models were fit for each SNP using PLINK version $1.07^{19}$, adjusted for 4 genomic principle components. SNP values were coded in terms of allele dosage. Fixed-effects meta-analyses were performed using R statistical software version 3.2.3, and $P$ $<5 \mathrm{E}-8$ was considered statistically significant.

Finally, we extracted results for several candidate SNPs/ genes that were previously implicated in $\mathrm{BE}$ or related phenotypes (bulimia nervosa spectrum), including $F T O$, $N T 5 C 1 B$, and $H T R 2 A^{23-25}$.

\section{Follow-up of GWAS results and gene set and network analysis}

GWAS results were annotated with NCBI Build 37 to determine location, nearby gene(s), and function. Top results were examined with the UCSC Genome Browser. Potential regulatory function was investigated using ENCODE data via the tools HaploReg (version v4.1 ${ }^{26}$ and RegulomeDB (version 1.1) ${ }^{27}$. Possible expression quantitative trait locus (eQTL) associations between the top SNPs and brain expression were explored with BRAINEAC data (http://www.braineac.org) ${ }^{28}$. For genomewide significant SNPs, we also explored potential regulatory mechanisms by visualizing 3D compartment organization in the region, based on potential chromatin looping identified with $\mathrm{Hi}-\mathrm{C}$ analysis, using the bioinformatics tool Juicebox ${ }^{29,30}$. Because large-scale folding organization is thought to be very stable across cell lines ${ }^{30}$, we visualized the 3D structure using the highest resolution dataset (data from the GM12878 lymphoblastoid cell line $\mathrm{e}^{30}$ ). Using the GTEX portal V6p $\mathrm{p}^{31}$, we also examined potential eQTL associations in brain and other tissues.

Case-only and case-control gene-level and gene-set analyses were conducted using MAGMA ${ }^{32}$. Gene-level tests were performed separately by study with multiple regression models using principal components of SNP effects computed from the imputed SNPs with dosage $\mathrm{R}^{2}$ $>0.8$ and $\mathrm{MAF}>0.01$. Gene-level meta-analysis was performed for 15,420 genes and utilized in the evaluation of $186 \mathrm{KEGG}$ pathways (http://www.genome.jp/kegg/ kegg1.html). Competitive test $p$-values were calculated and corrected for multiple testing via permutation.

Network analysis was performed with Ingenuity Pathway Analysis (IPA; www.ingenuity.com) using SNP $p$ values from the GWAS meta-analyses. SNPs were prefiltered using LD-clumping with $p \leq 0.05$, resulting in over 70,000 top SNPs considered for network analysis. SNPs were assigned to the nearest gene using NCBI Build 37, and the top 2000 genes (ranked by minimum SNP $p$ - value, all with $p<0.0002$ ) were considered 'significant' for network analysis. De-novo networks of direct and indirect interactions between significant genes in our data were constructed based on a functional analysis algorithm that utilizes published, peer-reviewed literature within the curated Ingenuity Knowledge Base.

\section{Results \\ GWAS results}

Supplemental Table 1 shows the sample demographics and supplemental Table 2 and 3 show the top results from the BE case-only GWAS of the GAIN and Mayo data, respectively. The Manhattan plot of the corresponding meta-analysis is shown in Fig. 1, with top results listed in Table 1. The meta-analysis of $\mathrm{BE}$ in $\mathrm{BD}$ cases provided genome-wide significant evidence for association of $\mathrm{BE}$ with a group of SNPs in the PRR5-ARHGAP8 gene, where the more common allele is associated with reduced risk of $\mathrm{BE}$ behavior among BD patients (top SNPs rs726170 and rs8139558, $\mathrm{OR}=0.52, p=3.05 \mathrm{E}-08)$; the $\mathrm{C}$ allele at rs726170 is less common in BD patients with BE (Mayo 0.84 , GAIN 0.81) as compared to those without BE (Mayo 0.89 , GAIN 0.90). Suggestive evidence of association with rs726170 in PRR5-ARHGAP8 was observed in our prior analysis of this phenotype in the GAIN data $(p=$ $8.0 \mathrm{E}-08) ;{ }^{11}$ the current analysis of the Mayo data replicated this finding $(p=0.0046)$, and the meta-analysis combining the results provided genome-wide significant evidence for association. Additionally, the meta-analysis showed suggestive evidence of association of the $G$ allele at rs7904579 in the $C U B N$ gene with $\mathrm{BE}$ behavior among $\mathrm{BD}$ patients $(\mathrm{OR}=1.56, p=1.4 \mathrm{E}-07)$, although this association was not genome-wide significant.

Supplemental Tables 4 and 5 show the top results from GWAS comparing cases with both BD and BE vs. non-BD controls in the GAIN and Mayo data, respectively. The Manhattan plot of the corresponding meta-analysis is shown in Fig. 2, with top results listed in Table 2.The top SNP rs111940429, which is genome wide significant $(P=$ $1.2 \mathrm{E}-08)$, is in an intergenic region of chromosome 2q12.3, with the closest gene PPP1R2P5; the $C$ allele is less common in BD patients with BE (Mayo 0.93, GAIN 0.93) than in controls (Mayo 0.96, GAIN 0.97).

\section{Regulatory analysis results}

The genome-wide significant SNP from our case-only meta-analysis, rs726170, is in high linkage disequilibrium (LD) with rs9614952 and rs28439052, which are situated at binding sites for transcription factor STAT3 (Fig. 3). It is also in complete LD with rs8139558, which is in an enhancer region of PRR5-ARHGAP8 with H3K4me1 histone mark in adipose cultures ${ }^{26}$. No significant eQTL associations between rs726170 and PRR5-ARHGAP8 expression were observed in human brain regions 
studied in BRAINEAC ${ }^{28}$. Using the GTEX portal (version V6p) ${ }^{31}$, rs726170 is a significant eQTL for ARHGAP8 in tibial nerve $(p=3.8 \mathrm{e}-9)$, but has no association with expression of the next $5^{\prime}$ gene $(P H F 21 B)$ or the next $3^{\prime}$ gene (PRR5).

Supplemental Fig. 1 shows the 3D genomic organization around rs726170. Yellow lines indicate block regions defined by marks of the transcription factor CCCTCbinding factor (CTCF), which delineates heterochromatin from euchromatin. This SNP is in the transition region between two blocks and shows little long range interactions, so it likely affects only the ARHGAP8 gene or another gene downstream (rather than genes $3^{\prime}$ of $A R H$ GAP8), consistent with the GTEX eQTL results. According to ENCODE data, this region is devoid of

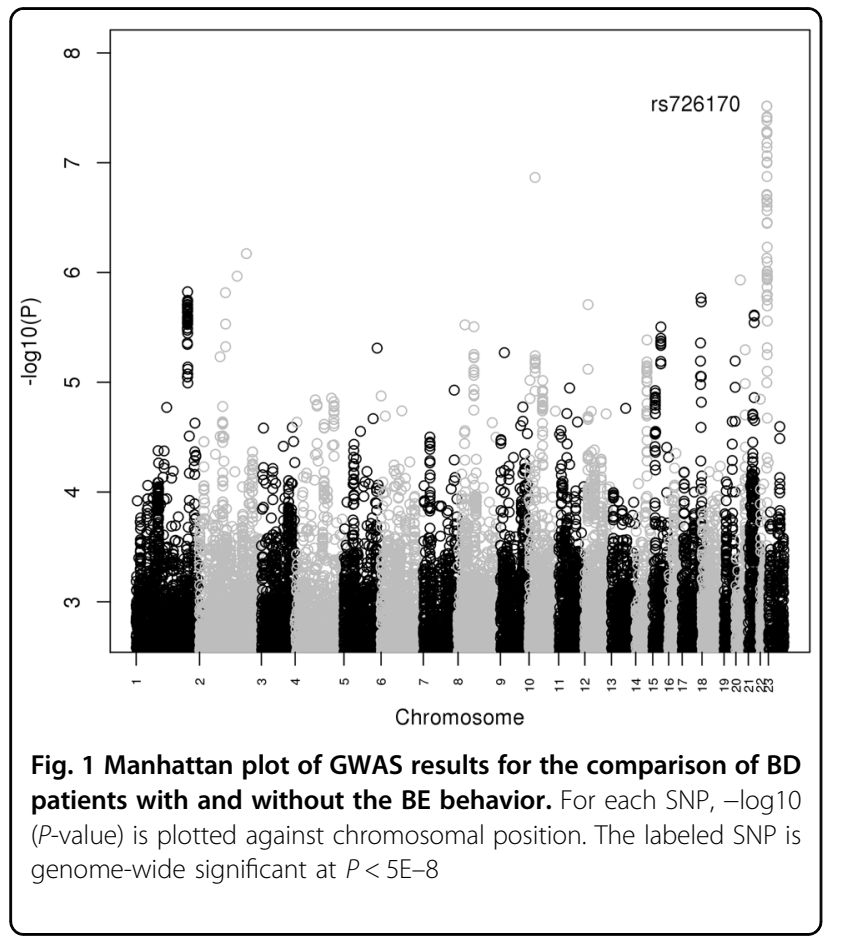

common histone marks in LCL tissues, with the exception for H3K9me3, normally associated with constitutively repressed genes.

Furthermore, rs111940429 identified in the case-control meta-analysis also shows evidence of regulatory function; specifically, the risk ('C') allele in our analysis potentially disrupts the motif for the transcription factor Fox ${ }^{26}$.

\section{Gene-set analysis and network analysis results}

MAGMA analyses did not identify any gene sets with statistically significant associations with BE after correction for multiple testing. The top-ranking gene sets in the case-only analysis and in the case-control analysis are shown in Supplemental Table 6 and Supplemental Table 7, respectively.

Constructed IPA networks focus on Neurological Disease, Psychological Disorders, Cellular Development (Supplemental Fig. 2), as well as Hereditary Disorders, Neurological Disease, and Organismal Injury and Abnormalities (Supplemental Fig. 3). Hub molecules with at least 5 connections in Network 1 (Supplemental Fig. 2) include WWOX, CUL3, CAND1, CDC5L, and SMARCA4. Hub molecules with at least 5 connections in Network 2 (Supplemental Fig. 3) include TADA2A, MED15, TCF7L2, $A T X N 1$, and RBFOX1.

\section{BE association with candidate genes}

We found no evidence of association of $\mathrm{BD}$ with comorbid BE, or BE in BD patients, with a set of SNPs/ genes selected based on prior human candidate gene studies (FTO, NT5C1B, and HTR2A). No SNP within these genes was statistically significant after Bonferroni correction for the number of candidate SNPs tested. The top result was rs7330461 in $H T R 2 A$ (uncorrected $p=$ 0.003 , corrected $p=0.90$ ).

\section{Discussion}

In this study, we utilized two BD case-control GWAS datasets to explore genetic risk for the sub-phenotype of

Table 1 Meta-analysis of BE in BD cases: top results

\begin{tabular}{|c|c|c|c|c|c|c|c|c|c|c|c|c|c|}
\hline SNP & Locus & Position & Gene & $\mathrm{A} 1$ & $\mathrm{~A} 2$ & $\begin{array}{l}\text { Mayo } \\
\text { A1 } \\
\text { freq }\end{array}$ & $\begin{array}{l}\text { GAIN } \\
\text { A1 } \\
\text { freq }\end{array}$ & Mayo OR & Mayo P & GAIN OR & GAIN P & $\begin{array}{l}\text { Meta- } \\
\text { analysis } \\
\text { OR }\end{array}$ & $\begin{array}{l}\text { Meta- } \\
\text { analysis } \\
p \text {-value }\end{array}$ \\
\hline rs726170 & $22 q 13.31$ & 45251811 & PRR5-ARHGAP8 & C & $\mathrm{T}$ & 0.88 & 0.88 & 0.62 & 0.0046 & 0.45 & $8.0 \mathrm{E}-07$ & 0.52 & $3.05 \mathrm{E}-08$ \\
\hline rs7904579 & 10p13 & 17131753 & CUBN & G & C & 0.35 & 0.37 & 1.62 & $6.9 \mathrm{E}-05$ & 1.50 & $4.9 \mathrm{E}-04$ & 1.56 & $1.36 \mathrm{E}-07$ \\
\hline rs1950038 & $2 q 32.1$ & 184444370 & intergenic & $T^{\prime}$ & C & 0.30 & 0.30 & 1.86 & $2.1 \mathrm{E}-06$ & 1.36 & 0.027 & 1.60 & $6.73 \mathrm{E}-07$ \\
\hline rs182107583 & $2 q 23.3$ & 150531537 & $\begin{array}{l}\text { LOC101929321 } \\
\text { (IncRNA) }\end{array}$ & A & C & 0.96 & 0.96 & 0.34 & 0.0015 & 0.29 & 2.0E-04 & 0.31 & $1.08 \mathrm{E}-06$ \\
\hline rs76087671 & 20p11.21 & 24311177 & intergenic & C & $\mathrm{T}$ & 0.94 & 0.95 & 0.61 & 0.0497 & 0.32 & $1.4 \mathrm{E}-06$ & 0.43 & 1.17E-06 \\
\hline
\end{tabular}

Note: Odds ratio (OR) estimates are presented in terms of $A 1$ vs. $A 2$ 


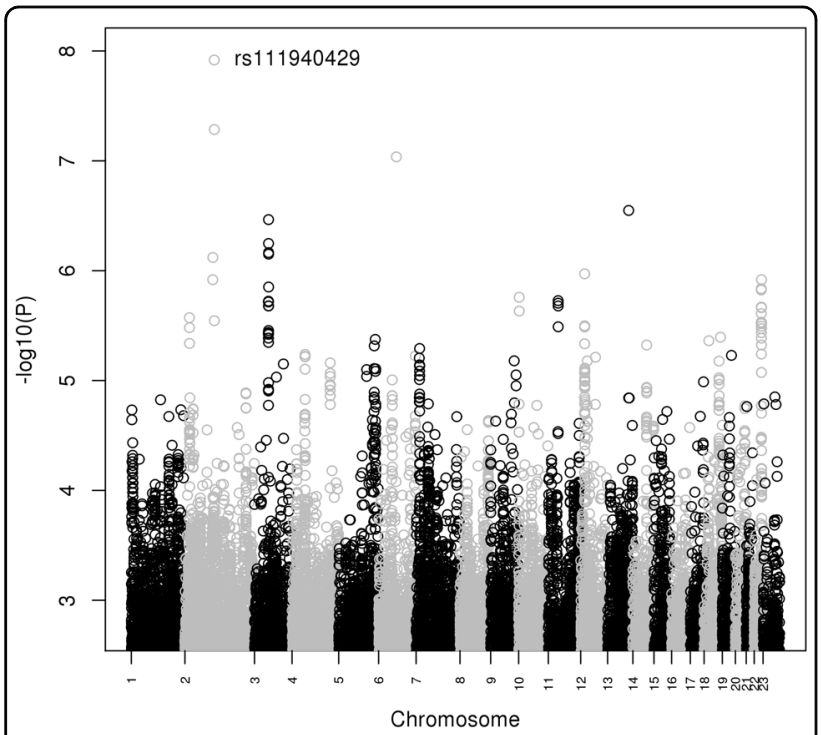

Fig. 2 Manhattan plot of GWAS results for the comparison of BD patients with the $\mathrm{BE}$ behavior sub-phenotype to controls. For each SNP, $-\log 10$ ( $P$-value) is plotted against chromosomal position. The labeled SNP is genome-wide significant at $P<5 \mathrm{E}-8$

$\mathrm{BD}$ with comorbid $\mathrm{BE}$. This is the largest GWAS of $\mathrm{BE}$ in $\mathrm{BD}$ to date, and no GWAS of BE in the general population has yet been published.

We demonstrated that $\mathrm{BE}$ among $\mathrm{BD}$ patients is associated with variants of the PRR5-ARHGAP8 gene, a readthrough transcript of neighboring genes PRR5 and $A R H$ GAP8. PRR5 is a circadian clock gene that encodes a subunit of the mammalian target of rapamycin complex 2 (mTORC2) critical for neuronal survival, proliferation, and differentiation ${ }^{33}$, as well as energy balance, obesity, and hyperphagia ${ }^{34}$. ARHGAP8 encodes a member of the large RhoGAP family of proteins, which are complex tissue-specific molecules regulated by lipid binding, protein-protein interactions, phosphorylation, and other mechanisms ${ }^{35}$. RhoGAPs mediate the cross-talk between Rho GTPases and other signaling pathways, and are fundamental to multiple cellular processes, such as cellular growth, gene transcription, and apoptosis ${ }^{35}$. Readthrough transcripts are fusions across adjacent genes resulting in proteins that share sequence identity with each individual gene product ${ }^{36}$. The PRR5-ARHGAP8 read-through occurs in nature ${ }^{37}$ and is expressed in the brain $^{31}$. Despite the unclear function of the PRR5ARHGAP8 fusion protein, $m$ TORC2 and Rho GTPases interact in different cellular processes, for example in regulating the cytoskeleton ${ }^{38}$, where this fusion protein may play a role.

Further information points to a potential regulatory role of rs726170, although the targets are unclear. This SNP is

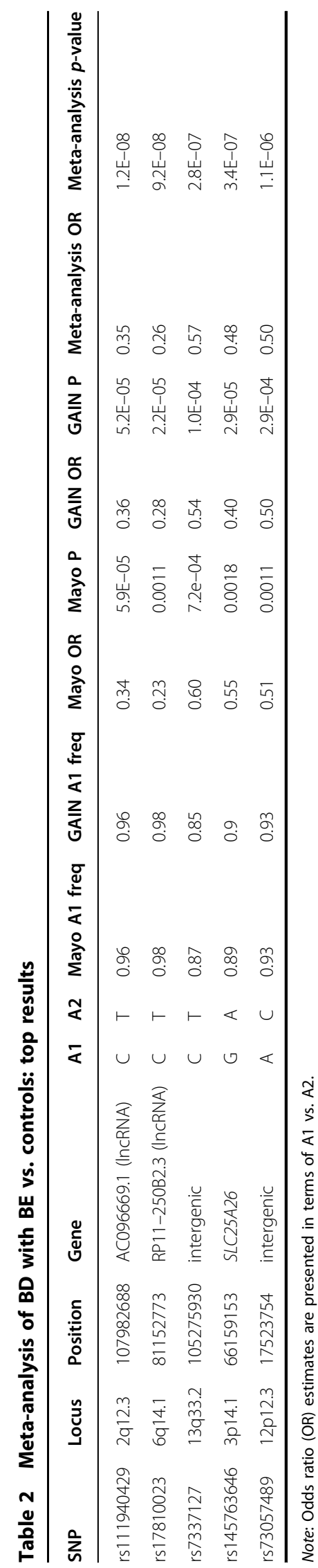




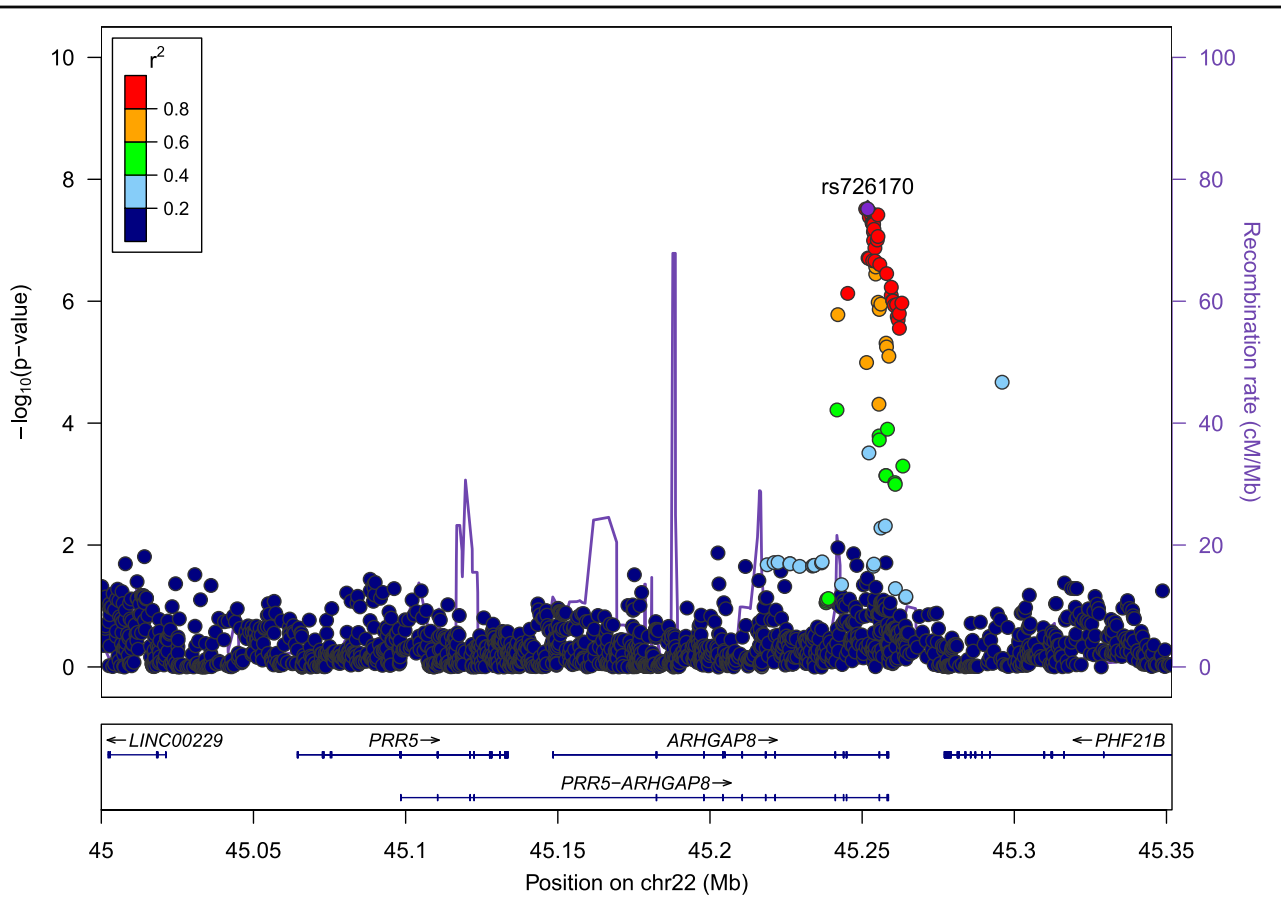

Fig. 3 Regional association plot of the ARHGAP8-PRR5 gene region, for association with BE in cases with BD

in high LD with SNPs in binding sites for the transcription factor STAT3 and an enhancer region in adipose tissue. While we have no evidence of this SNP being an eQTL for either PRR5 or ARHGAP8 in brain tissue, the variant is an eQTL for ARGHAP8 in tibial nerve tissue. Additionally, $\mathrm{Hi}-\mathrm{C}$ analysis suggested that the $3 \mathrm{D}$ chromatin structure of the region prohibits long-range interactions with genes that are upstream of the region, suggesting that the target is either ARHGAP8 or another gene downstream, rather than PRR5. ARHGAP8 is expressed at low levels in brain and blood, but high levels in gastrointestinal-related tissues. Given that rs726170 was not strongly associated with $\mathrm{BD}$ with comorbid $\mathrm{BE}$ compared to controls, collectively these results suggest that the identified SNP likely plays a role in $\mathrm{BE}$ rather than in $\mathrm{BD}$.

In the meta-analysis of $\mathrm{BD}$ cases with $\mathrm{BE}$ compared to controls, we identified a genome-wide significant variant in an intergenic region of chromosome $2 \mathrm{q} 12.3$, with the nearest gene encoding protein phosphatase 1 regulatory subunit 2 (PPP1R2), an inhibitor of the serine/threonine protein phosphatase 1 (PPP1) that binds to the active site of its catalytic subunit. The PPP1C/PPP1R2 complex has been shown to participate in neural functioning (calciuminduced synaptic scaling ${ }^{39}$, memory suppression $^{40}$, and neurodegeneration ${ }^{41,42}$ ), as well as mitosis and meiosis $^{43-46}$, cell morphology regulation ${ }^{47}$, and cardiac function ${ }^{48-50}$. PPP1R2 is expressed in GABAergic neurons in the striatum, cortex, and hippocampus ${ }^{51}$.
Our analyses revealed association trends for other genes and pathways that were not significant after multiple testing correction, but may nevertheless reflect important biological mechanisms. For example, the second top association signal in the case-only meta-analysis of $\mathrm{BE}$ among BD patients was in the gene encoding cubilin $(C U B N)$, a receptor for the intrinsic factor-vitamin $\mathrm{B} 12$ complex, which is essential for neurodevelopment; its deficiency has been implicated in various neuropsychiatric diseases such as depression, schizophrenia, dementia, and cognitive impairment ${ }^{52,53}$. In our MAGMA gene-set analysis comparing $\mathrm{BD}$ cases with $\mathrm{BE}$ to those without $\mathrm{BE}$, the canonical Wnt signaling pathway was a topranked pathway; this pathway is critical to neurogenesis, neurodevelopment ${ }^{54}$, mood stabilizer mechanism of action $^{55}$, and energy balance ${ }^{56}$. Application of IPA to our data produced a network that highlighted genes relevant for neurological and psychological disorders, with the effector of the canonical Wnt signaling pathway TCF7L2, a gene involved in genetic risk of BD with comorbid obesity $^{57,58}$, as a network hub (Supplemental Fig. 3). Finally, in our gene-set analysis of $\mathrm{BD}$ cases with $\mathrm{BE}$ compared to controls, the cytokine-cytokine receptor interaction pathway was among the top-ranking pathways; this finding is intriguing considering the role of cytokines in sickness response and the related suppression of appetite and induction of anhedonia. While possibly interesting, these results require further evidence from 
independent data before their role in binge eating can be established.

Although no prior GWAS of BE have been performed, a GWAS of anorexia nervosa identified a variant in SOX2$O T ;^{59}$ another variant in this region, rs4854912, was later confirmed in a GWAS of bipolar patients with either anorexia nervosa or bulimia nervosa compared to healthy controls, in an analysis that included GAIN data ${ }^{60}$. In our Mayo Biobank sample, we observed no evidence of association of this variant with $\mathrm{BE}$ among $\mathrm{BD}$ cases, nor in the comparison with control subjects (all $p>0.50$ ). However, because our phenotypes here focus on $\mathrm{BE}$ rather than anorexia nervosa or bulimia nervosa diagnoses, these differences in findings are not surprising. Candidate gene studies have suggested associations between binge eating and FTO in adolescents ${ }^{24}$, binge eating and HTR2A in young women ${ }^{23}$, and bulimia nervosa spectrum and $N T 5 C 1 B$ in female twins ${ }^{25}$, but we did not replicate any of these findings.

This study has several limitations. Without $\mathrm{BE}$ information in controls, it is not possible to determine whether the observed association with PRR5-ARHGAP8 reflects risk for $B E$ in general or risk for a subtype of $\mathrm{BD}$ with $\mathrm{BE}$. Additionally, $\mathrm{BE}$ was defined differently in the two data sets. In GAIN subjects, BE was defined as a lifetime history of food binges, or the rapid consumption of a large amount of food in a discrete period of time, usually less than two hours. In Mayo subjects, $\mathrm{BE}$ was defined as eating a large amount of food and having a sense of loss of control in the last six months. Nonetheless, rates of $\mathrm{BE}$ behavior were comparable in the two samples. Finally, the sample size was limited, resulting in low power to detect small and even moderate effects. By reducing phenotypic heterogeneity, which tends to result in increased power, we obtained genome-wide significant findings in this relatively small sample. Nevertheless, it is clear that much larger sample sizes are needed to identify further genetic risk factors for $\mathrm{BE}$.

In summary, this study provided genome-wide significant evidence for association of $\mathrm{BD}$ with comorbid $\mathrm{BE}$ with variants in the PRR5-ARHGAP8 region, and identified additional regions of interest in the etiology of $\mathrm{BD}$ with comorbid $\mathrm{BE}$. To date, $\mathrm{BD}$ genetic risk factors have been difficult to replicate ${ }^{61}$, due in part to extensive disease heterogeneity. The present work demonstrates the importance of reducing this heterogeneity by focusing on sub-phenotypes when investigating the genetic risk of $\mathrm{BD}$ and its comorbidities. Further population-based studies are needed to untangle whether the observed genetic association is driven by $\mathrm{BD}, \mathrm{BE}$, or both, and functional studies will be needed to determine the molecular targets and mechanisms behind the association.

\section{Data availability}

The GAIN dataset (part of Whole Genome Association Study of Bipolar Disorder) used for the analyses described in this manuscript were obtained from the database of Genotypes and Phenotypes (dbGaP) found at http://www. ncbi.nlm.nih.gov/gap through $\mathrm{dbGaP}$ accession number phs000017.v3.p1. The GTEx data used for the analyses described in this manuscript were obtained from: http:// www.gtexportal.org/home/bubbleHeatmapPage/

ARHGAP8 and http://www.gtexportal.org/home/gene/ ARHGAP8 on the GTEx Portal accessed on 30 Nov 2016.

\section{Acknowledgements}

This study was supported by funding from the Marriott Family Foundation and the Mayo Clinic Center for Individualized Medicine. Funding support for the Whole Genome Association Study of Bipolar Disorder was provided by the National Institute of Mental Health (NIMH) and the genotyping of samples was provided through the Genetic Association Information Network (GAIN). The Genotype-Tissue Expression (GTEx) Project was supported by the Common Fund of the Office of the Director of the National Institutes of Health. Additional funds were provided by the $\mathrm{NCl}, \mathrm{NHGRI}, \mathrm{NHLBI}, \mathrm{NIDA}, \mathrm{NIMH}$, and NINDS. Donors were enrolled at Biospecimen Source Sites funded by $\mathrm{NCl}$ / SAIC-Frederick, Inc. (SAIC-F) subcontracts to the National Disease Research Interchange (10XS170), Roswell Park Cancer Institute (10XS171), and Science Care, Inc. (X10S172). The Laboratory, Data Analysis, and Coordinating Center (LDACC) was funded through a contract (HHSN268201000029C) to The Broad Institute, Inc. Biorepository operations were funded through an SAIC-F subcontract to Van Andel Institute (10ST1035). Additional data repository and project management were provided by SAIC-F (HHSN261200800001E). The Brain Bank was supported by a supplement to University of Miami grants DA006227 and DA033684 and to contract N01MH000028. Statistical Methods development grants were made to the University of Geneva (MH090941 and MH101814), the University of Chicago (MH090951, MH090937, MH101820, MH101825), the University of North Carolina-Chapel Hill (MH090936 and MH101819), Harvard University (MH090948), Stanford University (MH101782), Washington University St Louis (MH101810), and the University of Pennsylvania (MH101822). The UK Brain Expression Consortium (BRAINEAC) was supported by the MRC through the MRC Sudden Death Brain Bank (C. S.), a Project Grant (G0901254 to J. H. and M. W.) and Training Fellowship (G0802462 to M. R.). D.T. was supported by the King Faisal Specialist Hospital and Research Centre, Saudi Arabia. Computing facilities used at King's College London were supported by the National Institute for Health Research (NIHR) Biomedical Research Centre based at Guy's and St Thomas' NHS Foundation Trust and King's College London.

\section{Author details}

${ }^{1}$ Lindner Center of HOPE, Mason, OH, USA. ${ }^{2}$ Department of Psychiatry and Behavioral Neuroscience, University of Cincinnati, Cincinnati, OH, USA. ${ }^{3}$ Department of Health Sciences Research, Mayo Clinic, Rochester, MN, USA. ${ }^{4}$ Department of Psychiatry, Universidad Autonoma de Nuevo Leon, Monterrey, Mexico. ${ }^{5}$ Department of Molecular Pharmacology and Experimental Therapeutics, Mayo Clinic, Rochester, MN, USA. ${ }^{6}$ University of Minnesota, Minneapolis, MN, USA. 'Department of Psychiatry and Psychology, Mayo Clinic, Rochester, MN, USA

\section{Conflict of interest}

Dr. S. L. Mc. E has been a consultant to or member of the scientific advisory board of Avanir, Bracket, F. Hoffmann-La Roche Ltd., MedAvante, Mitsubishi Tanabe Pharma Corporation, Myriad, Naurex, Novo Nordisk, Shire, and Sunovion. She has also been a principal or coinvestigatoron studies sponsored by Alkermes, Allergan, Azevan Pharmaceuticals, Forest, Marriott Foundation, Myriad, National Institute of Mental Health, Naurex, Novo Nordisk, Shire, Sunovion, and Takeda Pharmaceutical Company Limited. She is also an inventor on United States Patent No. 6,323,236 B2, Use of Sulfamate Derivatives for Treating Impulse ControlDisorders, and along with the patent's assignee, University of Cincinnati, Cincinnati, Ohio, hasreceived payments from Johnson and Johnson, which has exclusive rights under the patent. Dr. M. A. F. has 
received grant support from Assurex Health, Myriad, Pfizer, Marriott Foundationand Mayo Foundation; has been a consultant to Janssen Global Services, LLC, Mitsubishi Tanabe Pharma Corporation, Myriad, Sunovion, and Teva Pharmaceuticals; has received CME/Travel Support/presentation from CME Outfitters Inc. and Sunovion. The remaining authors declare that they have no conflict of interest.

\section{Publisher's note}

Springer Nature remains neutral with regard to jurisdictional claims in published maps and institutional affiliations.

Supplementary Information accompanies this paper at (https://doi.org/ 10.1038/s41398-017-0085-3)contains supplementary material.

Received: 13 June 2017 Revised: 6 September 2017 Accepted: 13 September 2017

Published online: 02 February 2018

\section{References}

1. Hudson, J. I., Hiripi, E., Pope, H. G. Jr. \& Kessler, R. C. The prevalence and correlates of eating disorders in the National Comorbidity Survey Replication. Biol. Psychiatry 61, 348-358 (2007).

2. Woldeyohannes, H. O. et al. Binge eating in adults with mood disorders: Results from the International Mood Disorders Collaborative Project. Obes. Res. Clin. Pract. 10, 531-543 (2015).

3. Castrogiovanni, S., Soreca, I., Troiani, D. \& Mauri, M. Binge eating, weight gain and psychosocial adjustment in patients with bipolar disorder. Psychiatry Res. 169, 88-90 (2009)

4. McElroy, S. L. et al. Clinical features of bipolar spectrum with binge eating behaviour. J. Affect. Disord. 201, 95-98 (2016).

5. Bulik, C. M., Sullivan, P. F. \& Kendler, K. S. Genetic and environmental contributions to obesity and binge eating. Int. J. Eat. Disord. 33, 293-298 (2003).

6. Lichtenstein, P. et al. Common genetic determinants of schizophrenia and bipolar disorder in Swedish families: a population-based study. Lancet $\mathbf{3 7 3}$ 234-239 (2009).

7. McGuffin, P. et al. The heritability of bipolar affective disorder and the genetic relationship to unipolar depression. Arch. Gen. Psychiatry 60, 497-502 (2003).

8. Root, T. L. et al. Shared and unique genetic and environmental influences on binge eating and night eating: a Swedish twin study. Eat. Behav. 11, 92-98 (2010).

9. Sullivan, P. F., Bulik, C. M. \& Kendler, K. S. Genetic epidemiology of binging and vomiting. Br. J. Psychiatry 173, 75-79 (1998).

10. Bould, H. et al. Parental mental illness and eating disorders in offspring. Int. J. Eat. Disord. 48, 383-391 (2015).

11. Winham, S. J. et al. Bipolar disorder with comorbid binge eating history: a genome-wide association study implicates APOB. J. Affect. Disord. 165 151-158 (2014)

12. Mailman, M. D. et al. The NCBI dbGaP database of genotypes and phenotypes. Nat. Genet. 39, 1181-1186 (2007)

13. Frye, M. A. et al. Development of a bipolar disorder biobank: differential phenotyping for subsequent biomarker analyses. Int. J. Bipolar Disord. 3, 30 (2015).

14. Olson, J. E. et al. The Mayo Clinic Biobank: a building block for individualized medicine. Mayo. Clin. Proc. 88, 952-962 (2013).

15. First M. B., Spitzer R. L., Gibbon M., Williams J. B. W. Structured Clinical Interview for DSM-IV-TR Axis I Disorders, Research Version, Patient Edition with Psychotic Screen (SCID-I/P WI PSY SCREEN). Biometric Research. (New York State Psychiatric Institute: New York, 2002)

16. Stice, E., Fisher, M. \& Martinez, E. Eating disorder diagnostic scale: additional evidence of reliability and validity. Psychol. Assess. 16, 60-71 (2004).

17. Stice, E., Telch, C. F. \& Rizvi, S. L. Development and validation of the eating disorder diagnostic scale: a brief self-report measure of anorexia, bulimia, and binge-eating disorder. Psychol. Assess. 12, 123-131 (2000).

18. Krabbenborg, M. A. et al. The eating disorder diagnostic scale: psychometric features within a clinical population and a cut-off point to differentiate clinical patients from healthy controls. Eur. Eat. Disord. Rev. 20, 315-320 (2012).
19. Smith, E. N. et al. Genome-wide association study of bipolar disorder in European American and African American individuals. Mol. Psychiatry 14, 755-763 (2009).

20. Pritchard, J. K. Stephens, M. \& Donnelly, P. Inference of population structure using multilocus genotype data. Genetics 155, 945-959 (2000).

21. Delaneau, O., Marchini, J. \& Zagury, J. F. A linear complexity phasing method for thousands of genomes. Nat. Methods 9, 179-181 (2012).

22. Howie, B., Fuchsberger, C., Stephens, M., Marchini, J. \& Abecasis, G. R. Fast and accurate genotype imputation in genome-wide association studies through pre-phasing. Nat. Genet. 44, 955-959 (2012).

23. Koren, $R$. et al. Preliminary evidence for the role of HTR2A variants in binge eating in young women. Psychiatr. Genet. 24, 28-33 (2014).

24. Micali, N., Field, A. E., Treasure, J. L. \& Evans, D. M. Areobesity risk genes associated with binge eating in adolescence? Obesity 23, 1729-1736 (2015).

25. Wade, T. D. et al. Genetic variants associated with disordered eating. Int. J. Eat Disord. 46, 594-608 (2013)

26. Kheradpour, P. \& Kellis, M. Systematic discovery and characterization of regulatory motifs in ENCODE TF binding experiments. Nucleic Acids Res. 42 2976-2987 (2014)

27. Boyle, A. P. et al. Annotation of functional variation in personal genomes using RegulomeDB. Genome Res. 22, 1790-1797 (2012).

28. Trabzuni, D. et al. Quality control parameters on a large dataset of regionally dissected human control brains for whole genome expression studies. J. Neurochem. 119, 275-282 (2011).

29. Durand, N. C. et al. Juicebox provides a visualization system for Hi-C contact maps with unlimited zoom. Cell. Syst. 3, 99-101 (2016).

30. Rao, S. S. et al. A 3D map of the human genome at kilobase resolution reveals principles of chromatin looping. Cell 159, 1665-1680 (2014).

31. GTEx Consortium.. Human genomics. The Genotype-Tissue Expression (GTEx) pilot analysis: multitissue gene regulation in humans. Science $\mathbf{3 4 8}, 648-660$ (2015).

32. de Leeuw, C. A., Mooij, J. M., Heskes, T. \& Posthuma, D. MAGMA: generalized gene-set analysis of GWAS data. PLoS. Comput. Biol. 11, e1004219 (2015)

33. Wahane, S. D. et al. PI3K-p110-alpha-subtype signalling mediates survival, proliferation and neurogenesis of cortical progenitor cells via activation of mTORC2. J. Neurochem. 130, 255-267 (2014).

34. Kocalis, H. E. et al. Rictor/mTORC2 facilitates central regulation of energy and glucose homeostasis. Mol. Metab. 3, 394-407 (2014).

35. Tcherkezian, J. \& Lamarche-Vane, N. Current knowledge of the large RhoGAP family of proteins. Biol. Cell. 99, 67-86 (2007)

36. Akiva, P. et al. Transcription-mediated gene fusion in the human genome Genome Res. 16, 30-36 (2006).

37. O'Leary, N. A. et al. Reference sequence (RefSeq) database at NCBI: current status, taxonomic expansion, and functional annotation. Nucleic Acids Res. $\mathbf{4 4}$ D733-D745 (2016)

38. Bracho-Valdés, l. et al. mTORC1- and mTORC2-interacting proteins keep their multifunctional partners focused. lubmb. Life. 63, 896-914 (2011).

39. Siddoway, B. A. et al. An essential role for inhibitor-2 regulation of protein phosphatase-1 in synaptic scaling. J. Neurosci. 33, 11206-11211 (2013).

40. Yang, H. et al. Protein Phosphatase-1 Inhibitor-2 Is a novel memory suppressor J. Neurosci. 35, 15082-15087 (2015)

41. Tanimukai, H., Grundke-lqbal, I. \& lqbal, K. Up-regulation of inhibitors of protein phosphatase-2A in Alzheimer's disease. Am. J. Pathol. 166, 1761-1771 (2005).

42. Arif, M. et al. Cytoplasmic retention of protein phosphatase $2 \mathrm{~A}$ inhibitor 2 (I2PP2A) induces Alzheimer-like abnormal hyperphosphorylation of Tau. J. Biol. Chem. 289, 27677-27691 (2014).

43. Ceulemans, H. \& Bollen, M. Functional diversity of protein phosphatase-1, a cellular economizer and reset button. Physiol. Rev. 84, 1-39 (2004).

44. Leach, C., Shenolikar, S. \& Brautigan, D. L. Phosphorylation of phosphatase inhibitor-2 at centrosomes during mitosis. J. Biol. Chem. 278, 26015-26020 (2003).

45. Wang, W. Stukenberg, P. T. \& Brautigan, D. L. Phosphatase inhibitor-2 balances protein phosphatase 1 and aurora B kinase for chromosome segregation and cytokinesis in human retinal epithelial cells. Mol. Biol. Cell. 19, 4852-4862 (2008).

46. Wang, $X$. et al. Endogenous regulators of protein phosphatase-1 during mouse oocyte development and meiosis. Reproduction 128 493-502 (2004).

47. Terry-Lorenzo, R. T. et al. Neurabins recruit protein phosphatase-1 and inhibitor-2 to the actin cytoskeleton. J. Biol. Chem. 277, 46535-46543 (2002). 
48. Grote-Wessels, S. et al. Inhibition of protein phosphatase 1 by inhibitor-2 exacerbates progression of cardiac failure in a model with pressure overload. Cardiovasc. Res. 79, 464-471 (2008).

49. Kirchhefer, U. et al. Enhanced cardiac function in mice overexpressing protein phosphatase Inhibitor-2. Cardiovasc. Res. 68, 98-108 (2005).

50. Yamada, M. et al. Inhibition of protein phosphatase 1 by inhibitor-2 gene delivery ameliorates heart failure progression in genetic cardiomyopathy. Faseb. J. 20, 1197-1199 (2006).

51. Belforte, J. E. et al. Postnatal NMDA receptor ablation in corticolimbic inter neurons confers schizophrenia-like phenotypes. Nat. Neurosci. 13, 76-83 (2010).

52. Bottiglieri, T. Folate, vitamin B12, and neuropsychiatric disorders. Nutr. Rev. 54, 382-390 (1996)

53. Zhang, Y. et al. Decreased Brain Levels of Vitamin B12 in Aging, Autism and Schizophrenia. PLoS. One 11, e0146797 (2016).

54. Kim, W. Y. \& Snider, W. D. Functions of GSK-3 Signaling in Development of the Nervous System. Front. Mol. Neurosci. 4, 44 (2011).
55. Klein, P. S. \& Melton, D. A. A molecular mechanism for the effect of lithium on development. Proc. Natl. Acad. Sci. USA 93, 8455-8459 (1996).

56. Helfer, G. \& Tups, A. Hypothalamic Wnt Signalling and its Role in Energy Balance Regulation. J. Neuroendocrinol. 28, 12368 (2016).

57. Cuellar-Barboza, A. B. et al. Accumulating evidence for a role of TCF7L2 variants in bipolar disorder with elevated body mass index. Bipolar Disord. 18, 124-135 (2016).

58. Winham, S. J. et al. Genome-wide association study of bipolar disorder accounting for effect of body mass index identifies a new risk allele in TCF7L2 Mol Psychiatry. 19, 1010-1016 (2014).

59. Boraska, V. et al. A genome-wide association study of anorexia nervosa. Mol. Psychiatry 19, 1085-1094 (2014).

60. Liu, X. Bipolar Genome S, Kelsoe JR, Greenwood TA. A genome-wide association study of bipolar disorder with comorbid eating disorder replicates the SOX2-OT region. J. Affect. Disord. 189, 141-149 (2016).

61. Psychiatric GWAS Consortium Bipolar Disorder Working Group. Large-scale genome-wide association analysis of bipolar disorder identifies a new susceptibility locus near ODZ4. Nat. Genet. 43, 977-983 (2011). 